\title{
The Immune Deficiency and Dysregulation Activity (IDDA2.1 'Kaleidoscope') Score and Other Clinical Measures in Inborn Errors of Immunity
}

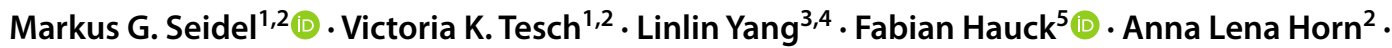 \\ Maria Anna Smolle ${ }^{6}$. Franz Quehenberger ${ }^{7}$ (D) Martin Benesch ${ }^{1}$
}

Received: 10 June 2021 / Accepted: 25 October 2021 / Published online: 19 November 2021

(c) The Author(s) 2021, corrected publication 2022

\begin{abstract}
Quantifying the phenotypic features of rare diseases such as inborn errors of immunity (IEI) helps clinicians make diagnoses, classify disorders, and objectify the disease severity at its first presentation as well as during therapy and follow-up. Furthermore, it may allow cross-sectional and cohort comparisons and support treatment decisions such as an evaluation for transplantation. On the basis of a literature review, we provide a descriptive comparison of ten selected scores and measures frequently used in IEI and divide these into three categories: (1) diagnostic tools (for Hyper-IgE syndrome, hemophagocytic lymphohistiocytosis, and Wiskott-Aldrich syndrome), (2) morbidity and disease activity measures (for common variable immune deficiency [CVID], profound combined immune deficiency, CTLA-4 haploinsufficiency, immune deficiency and dysregulation activity [IDDA], IPEX organ impairment, and the autoinflammatory disease activity index), and (3) treatment stratification scores (shown for hypogammaglobulinemia). The depth of preclinical and statistical validations varies among the presented tools, and disease-inherent and user-dependent factors complicate their broader application. To support a comparable, standardized evaluation for prospective monitoring of diseases with immune dysregulation, we propose the IDDA2.1 score (comprising 22 parameters on a 2-5-step scale) as a simple yet comprehensive and powerful tool. Originally developed for use in a retrospective study in LRBA deficiency, this new version may be applied to all IEI with immune dysregulation. Reviewing published aggregate cohort data from hundreds of patients, the IDDA kaleidoscope function is presented for 18 exemplary IEI as an instructive phenotype-pattern visualization tool, and an unsupervised, hierarchically clustered heatmap mathematically confirms similarities and differences in their phenotype expression profiles.
\end{abstract}

Keywords Inborn error of immunity (IEI) · primary immunodeficiency (PID) $\cdot$ primary immune regulatory disorder (PIRD) $\cdot$ score $\cdot$ scale $\cdot$ measure $\cdot$ index $\cdot$ algorithm $\cdot$ heatmap $\cdot$ spider chart

Markus G. Seidel

markus.seidel@medunigraz.at

1 Division of Pediatric Hematology-Oncology, Department of Pediatrics and Adolescent Medicine, Medical University of Graz, Auenbruggerplatz 38, A-8036 Graz, Austria

2 Research Unit for Pediatric Hematology and Immunology, Medical University of Graz, Graz, Austria

3 Department of Clinical Immunology, Royal Free London NHS Foundation Trust, London NW3 2PF, UK

4 Institute for Immunity and Transplantation, University College London, London NW3 2PF, UK
5 Division of Pediatric Immunology and Rheumatology, Department of Pediatrics, Dr. Von Hauner Children's Hospital, University Hospital, Ludwig-Maximilians-Universität München, Munich, Germany

6 Department of Orthopedics and Trauma, Medical University of Graz, Graz, Austria

7 Institute for Medical Informatics, Statistics, and Documentation, Medical University Graz, Graz, Austria 


\section{Abbreviations}

AI-cytopenia

AIDAI

AIS

APDS

\section{CARD11-GOF}

CHAI

CNS

CTLA-4

CVID

DEF6

DOCK8

GLILD

HIES

HLH

HPO

IBD

ICU

IDDA score

\section{IDDM}

IEI

IPEX syndrome

\section{IUIS}

IVIG, SCIG

LIP

LRBA

NFKB1/NFKB2

NK cells

P-CID

PID

PIK3CD / PIK3R1
Autoimmune cytopenia

Autoinflammatory disease activity index

Autoinflammatory syndrome Activated phosphoinositide

3-kinase $\delta$ syndrome

Caspase recruitment domaincontaining protein 11 gain-of function

CTLA-4 insufficiency with autoimmune infiltration

Central nervous system

Cytotoxic T-lymphocyte

antigen-4

Common variable

immunodeficiency

Differentially expressed in

FDCP 6 homolog

Dedicator of cytokinesis 8

Granulomatous lymphocytic interstitial lung disease

Hyper-IgE syndrome

Hemophagocytic

lymphohistiocytosis

Human phenotype ontology

Inflammatory bowel disease

Intensive care unit

Immune deficiency and dysregulation activity score

Insulin-dependent diabetes

mellitus

Inborn error of immunity

Immunodeficiency polyen-

docrinopathy enteropathy,

$\mathrm{X}$-linked syndrome

International Union of

Immunological Societies

Intravenous or subcutaneous

immunoglobulin $\mathrm{G}$ therapy

Lymphocytic interstitial

pneumonitis

Lipopolysaccharide-

responsive beige-like anchor

protein

Nuclear factor kappa B1/2

Natural killer cells

Profound combined

immunodeficiency

Primary immunodeficiency

Phosphoinositide 3-kinase

genes
PIRD

PRKCD
SCID

Primary immune regulatory

disorder

Protein kinase $\mathrm{C}$ delta

Severe combined

immunodeficiency

STAT3-LOF / STAT1-LOF Signal transducer and activator of transcription-3 (-1)

loss-of-function

TET2

Ten eleven translocation

(TET) methylcytosine dioxygenase 2

Treg cells

Tregopathies

WAS

XIAP, XLP2 (gene

XLP1 (gene

Regulatory T cells

Regulatory $\mathrm{T}$ cell disorders

Wiskott-Aldrich syndrome

BIRC4): X-linked inhibitor

of apoptosis

SH2D1A): X-linked lym-

phoproliferative syndrome

type 1

\section{Introduction}

In clinical oncology and rheumatology, staging, grading, and diagnostic criteria are routinely applied to define the diagnosis or a subtype of a disorder and consequently to determine the appropriate management of a patient and the treatment of a disease. Similarly, scores that include physiological, biometrical, laboratory and medical history data are applied to assess the probability of survival of patients in intensive care units (broadly or more or less organ-specific) or to quantify end-stage organ damage, e.g., liver disease. However, in the immunodeficiency clinic, such algorithms and guidelines are less frequently available. Most patients with IEI are managed on a descriptive-diagnosis and problemdirected - more or less individual — basis, with the exception of patients with severe combined immunodeficiency (SCID) and certain other distinct inborn errors of immunity (IEI), where a clear genotype-phenotype correlation and the therapeutic consequences of a (mostly genetic) diagnosis (e.g., a targeted therapy, gene therapy, or hematopoietic stem cell transplantation [HSCT]) have been established. This management is especially challenging in immunodeficient patients with accompanying syndromic or complex features, such as immune dysregulation with autoimmunity and inflammation, a predisposition for malignancy, bleeding, or hemophagocytosis, or other increased risks. To categorize IEI phenotypes into most likely (sub-)diagnoses, document the natural clinical course of the disease, evaluate treatment responses, and scientifically improve management recommendations for these individuals, various attempts have been made to quantify and standardize the assessment of a disease 
phenotype and category by using scores, indexes, scales, and other measures.

In the first part, we provide an overview of various types of clinical scores currently used in IEI. Without claiming to be exhaustive, we briefly describe ten representative tools and compare their scopes, applications, and limitations. The second part presents an updated version of the immune deficiency and dysregulation activity (IDDA) score. Originally developed to perform a retrospective comparison of the clinical courses and disease burden of patients with a deficiency of the lipopolysaccharide and beige-like anchor protein (LRBA)[1] to compare long-term outcomes with or without HSCT, the presented version IDDA2.1 is designed to be a useful measure for prospectively assessing the immune deficiency and dysregulation activity of all patients with IEI and immune dysregulation, including primary immune regulatory disorders (PIRDs) as well as many combined immunodeficiencies and predominantly antibody deficiencies (categories I-IV of the IEI classification of the International Union of Immunological Societies [IUIS] [2, 3]). We demonstrate the power of its parameters by using unsupervised hierarchical clustering across various IEI with immune dysregulation and discuss advantages of the IDDA2.1 score, particular differences as compared to other measures in IEI, and the future potential as a predictive tool for disease (sub-)categorization, allowing for the deduction of specific screening recommendations on the basis of those complications expected for the respective IEI, as well as serving as a guide to support phenotype-targeted diagnostic and treatment decisions in patients without a genetic diagnosis and monitoring their effects. Finally, the kaleidoscope function of the IDDA2.1 score is applied to 18 IEI with immune dysregulation of various IUIS categories, demonstrating its educational value.

\section{Routine Applications and Brief Description of Representative Types of Clinical Scores in IEI}

On the basis of a literature review performed in January 2021, we selected ten representative instruments that are used as measures, scores, scales, or indexes (hereafter generally referred to as "scores") to assess clinical features and the disease activity of IEI quantitatively and qualitatively. Among these frequently used tools, we defined three categories of clinical scores for IEI with different fields of applications and consequently varying sets of parameters (Table 1 , Fig. 1). These include the following:

1) Diagnostic scores comprising a set of disease-specific weighted criteria can increase the likelihood of making a specific diagnosis or sub-classifying a diagnosis.
Three examples of tools assisting diagnosis-making are the Hyper-IgE syndrome (HIES) score, which was originally designed for autosomal dominant STAT3-LOF syndrome [4, 5], and the 'diagnostic guidelines' and the 'H score' used for primary and secondary hemophagocytic lymphohistiocytosis (HLH), respectively [6-8] (Table 1). These are widely accepted and statistically validated tools that are used to corroborate making a diagnosis of a suspected IEI or defining the likelihood of the presence of the respective syndrome. Of note, these measures are not designed to guide a differential diagnostic work-up in presence of only one or few of their predominant symptoms. Their examples, however, illustrate the challenges associated with defining an entity by applying clinical diagnostic criteria independently of the existence/awareness of a genetic background. Namely, the definition of the HIES of genetic origins other than mutations in STAT3 or differences between the familial or acquired forms of hemophagocytic syndromes required modifications of the original definitions. For instance, in the case of deficiency of DOCK8, the HIES score was adapted to reflect additional and unique features of this other type of HIES [9]. While the HIES score is predominantly based on preexisting or aggravating anatomical features in an individual, their infectious history and laboratory values, and includes a correction for a young age, the hemophagocytosis scores rely mainly on laboratory values, fever, and organomegaly (Table 1, Fig. 1). The diagnostic tool for familial hemophagocytic lymphohistiocytosis was defined by the Histiocyte Society [8], and differs slightly from other sets of diagnostic criteria that have been proposed for secondary HLH $[6,10,11]$, as well as from the $\mathrm{H}$ score for acquired HLH, which is also applied to correct for potentially causative or modifying factors, such as underlying malignancy, infection, or existing pharmacological immunosuppression [7].

An instrument used to classify a subcategory of a diagnosis by taking into account the presence of certain features or complications can help to define the subtype of an IEI. The Wiskott-Aldrich syndrome (WAS) score is a typical example of the instruments that are used to categorize a diagnosis into subtypes [12-14]. In this $\mathrm{X}$-linked syndrome, typically characterized by the triad of infection, microthrombocytopenia, and eczema, the phenotypic spectrum covers a wide range that usually does not change over time within one patient. This spectrum includes "classic/severe" WAS \pm autoimmunity categories, various categories in X-linked thrombocytopenia (intermittent or persistent, \pm eczema and immunodeficiency) and in X-linked neutropenia (mildest form) on a scale ranging from 0 to 5. Although classifying patients from mild to severe forms of WAS and used in 
Journal of Clinical Immunology (2022) 42:484-498

487

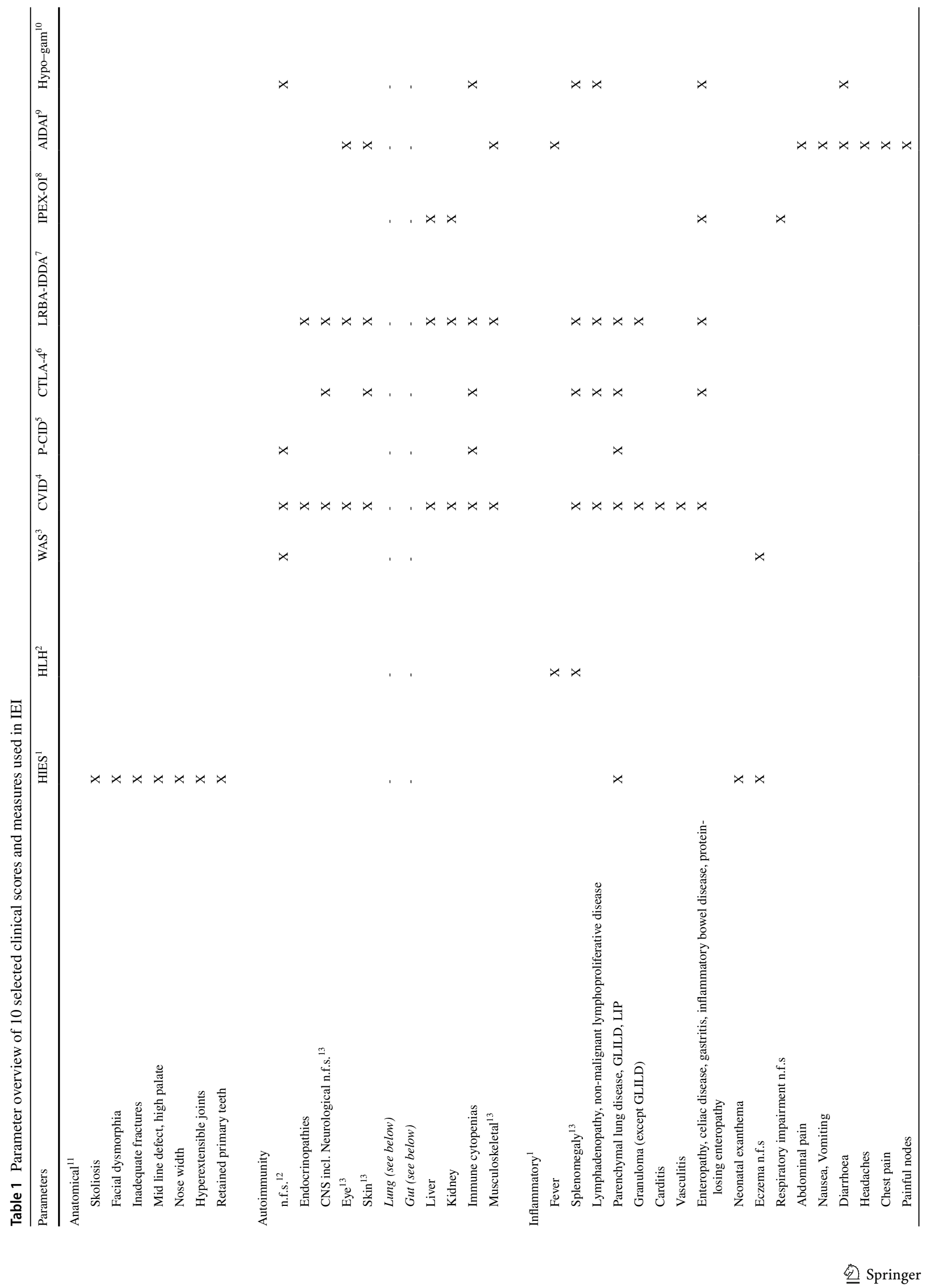




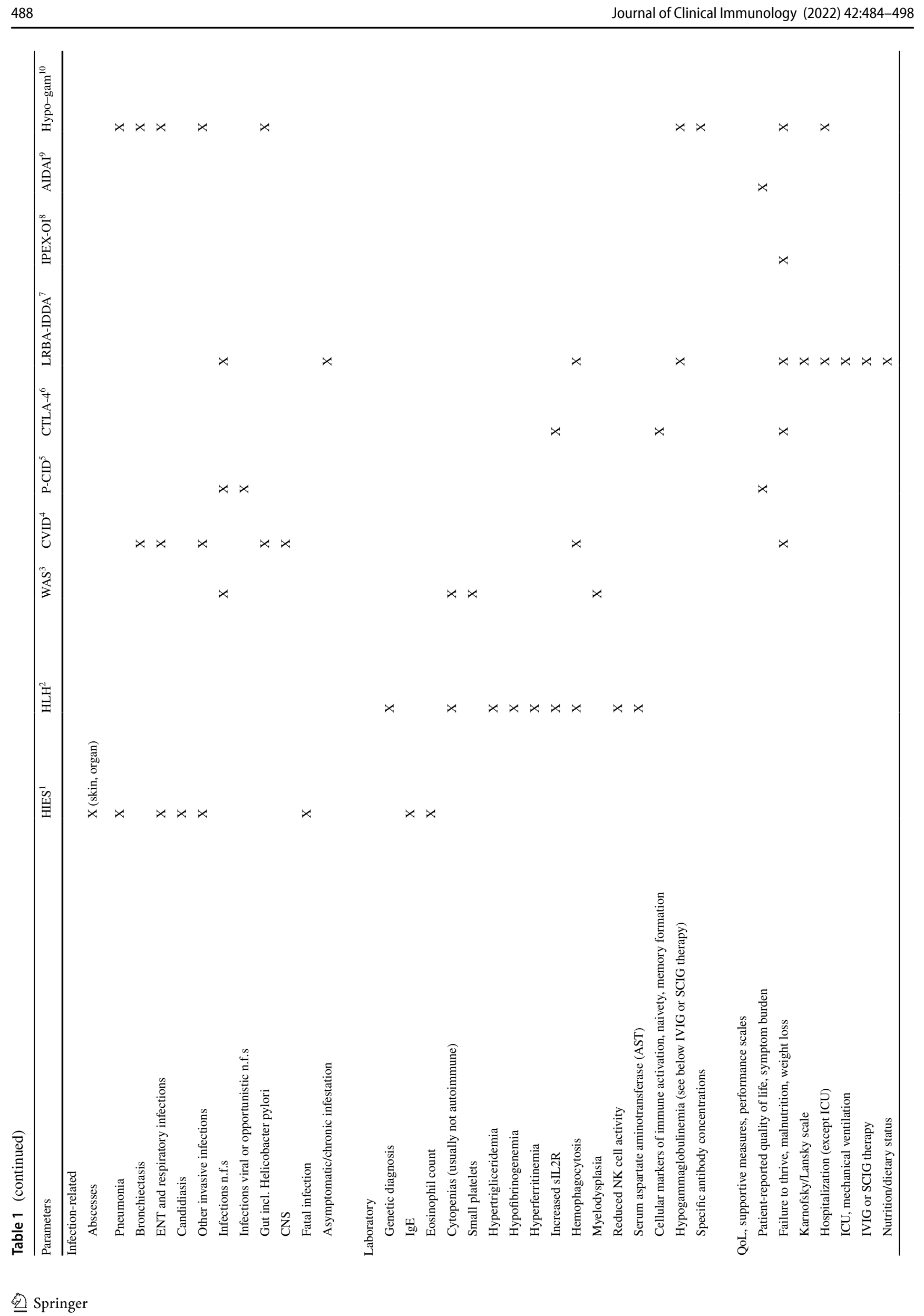




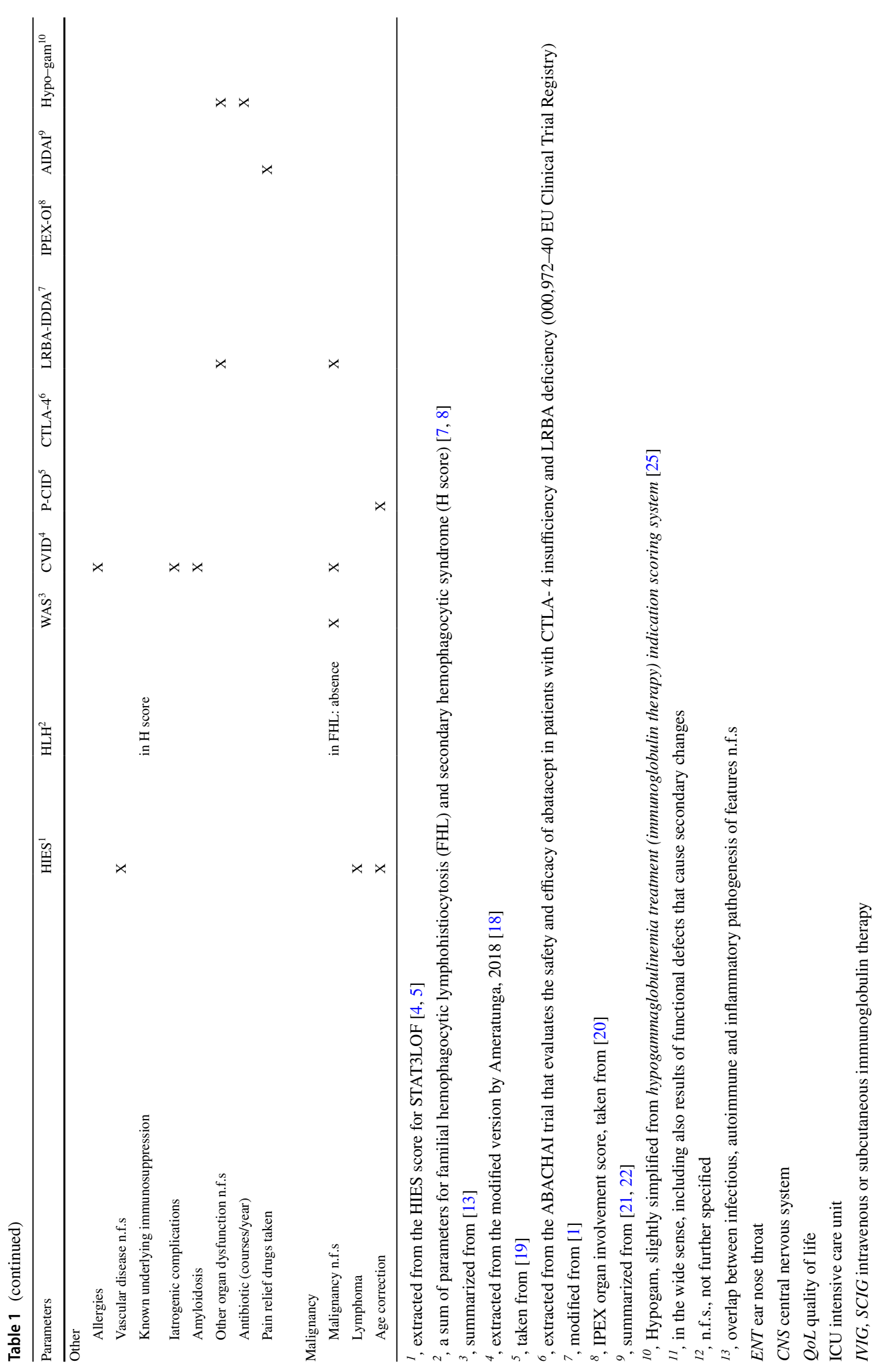




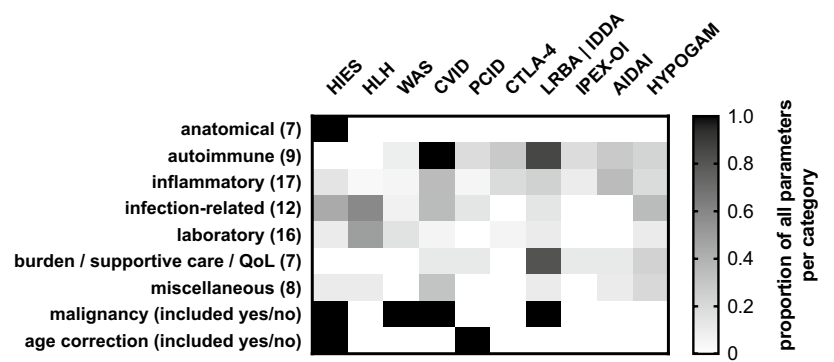

Fig. 1 Schematic comparison of scores and measures routinely used in the IEI clinic. Please refer to Table 1 and the original publications for more details. The selection of scores represents an exemplary choice of the authors

clinical cohort studies, this score has been shown to be of relatively little predictive relevance with regard to the development of autoimmunity or malignancy [15].

2) Morbidity and disease activity measures can be applied to grade the disease severity and burden, which may change during the clinical course of one patient. These scores are typically re-assessed at every clinical visit or scheduled time points of a patient with a defined IEI. Different results for these scores may reflect a separate subtype of the disorder or, more often, a distinct phase of the disease the patient is going through. One example is the collection of common variable immune deficiency (CVID) or CVID complication scores [16-18]; these scores can be used to quantify the severity of organ involvement and the accumulating inflammatory or autoimmune and even treatment-related complications in a patient. Points are added for every feature, and the scores are observed over the patient history. For profound combined immunodeficiency (P-CID), a morbidity measure was introduced that is part of an ongoing prospective study, which includes bacterial, viral, and opportunistic infections, immune-mediated cytopenia or other signs of immune dysregulation, and lung involvement, and corrects for the time of exposure (number of events divided by patient's age); additional information on the patientreported quality of life is analyzed separately [19]. A more disease-specific score than those used for CVID or P-CID is the CTLA-4 haploinsufficiency with autoimmune infiltration (CHAI) morbidity measure, which is being used in the ongoing abatacept-CHAI study ( $B$. Grimbacher, personal communication). The CHAI morbidity measure quantifies the organ involvement in detail by translating specific lab values including results of FACS analyses, imaging studies, or physiological functional results into points. The item list includes many non-routine investigations and thus requires a structured patient visit with all tests to be planned beforehand. A related disease with many features similar to CTLA-4 haploinsufficiency is LRBA deficiency, for which we recently developed a simple but comprehensive score that includes both a quantification of the severity of infectious complications and of features of immune dysregulation, and the need for supportive care, performance scale, hospitalization days, IgG substitution therapy, and nutritional interventions to reflect the entire disease burden [1]. This score with its differences from other presented measures, named immune deficiency and dysregulation activity (IDDA) score, is explained in more detail below. Another, even much simpler but effective, disease-specific score is the assessment of organ involvement in IPEX syndrome (immune dysregulation, polyendocrinopathy, enteropathy, $\mathrm{X}$-linked) on a scale from 0 to 5 ( 1 point per prognosis-relevant organ involvement) [20]. This score was used as part of a highly thorough and comprehensive retrospective analysis of patient- and treatment-related factors in IPEX syndrome and was identified as being significantly linked to the HSCT outcome of these patients [20].

Assessing the disease severity and burden is also an important factor in the management of patients with autoinflammatory syndromes (AIS) such as hereditary fever syndromes. The autoinflammatory disease activity index (AIDAI) represents a different type of tool based on a patient-maintained disease diary that was developed and statistically validated in a multistep process; this index has been designed to suit many of the known AIS $[21,22]$, a fact that is corroborated by its application, even if in slightly adapted forms, in current clinical trials $[23,24]$.

3) Finally, treatment stratification scoring systems may be used to support decisions on therapy. In IEI, a widely relevant example is the score to guide the initiation of immunoglobulin therapy based on laboratory parameters and the clinical history of adult patients with hypogammaglobulinemia (including CVID; Table 1 and Fig. 1) [25]. With a similar aim, a more complex version of a treatment algorithm for patients with primary antibody deficiencies was published as interdisciplinary consensus- and evidence-based guideline by the Association of Scientific Medical Societies in Germany (AWMF) and updated recently $[26,27]$. Although the latter instrument includes many similar criteria extracted from the history, clinical status, and laboratory parameters of hypogammaglobulinemic patients like the above-mentioned score, it was not designed as a 'score' but as a step-bystep algorithm that includes degrees of evidence for each criterion.

The proportional comparison of items and parameter categories used in each of these ten scores are depicted in Fig. 1 and listed in Table 1 (the original publication of each 
of the selected representative scores is cited for reference and to provide access to more detailed information). While type 1 scores from the list above rely on yes/no criteria or a semiquantitative "staging" to support making a diagnosis, most of the type 2 and 3 scores are supposed to be applied repeatedly to grade the actual disease activity status of a patient by adding scores for the affected organs, symptoms, complications, or other features. Some of these scores can even quantify the severity of each criterion. The latter quality of a score is needed if it should serve as a monitoring tool to document the clinical course of a patient in longitudinal studies, e.g., in prospective drug trials or for registry purposes. Naturally, the extent and application of a score in IEI reflects the difficulties associated with making either a diagnosis or managing affected patients with a certain disorder. Thus, using the established scores in the routine management of patients with IEI has a practical and beneficial awareness-raising effect, i.e., to prevent mistakes and negligence when applying the recommended monitoring measures, as the respective scores are often based on the experience of large patient series that encompass the full phenotypic spectrum of a rare disease known at that time.

\section{The Immune Deficiency and Dysregulation Activity (IDDA2.1 'Kaleidoscope') Score}

The IDDA2.1 score is designed as a simple, physicianreported outcome measure tool and is used to assess the severity of the involvement of 12 organ systems in immune dysregulation (e.g., autoimmunity, autoinflammation, lymphoproliferation, granuloma formation) and two other clinical features (failure to thrive and severe infections) that are scored on a 5 -step scale from 0 to 4 (indicating $0=$ absent; $1=$ mild, transient, not requiring treatment; $2=$ moderate, intermittent therapy needed; $3=$ severe, continuous therapy needed; $4=$ life-threatening, refractory, irreversible; Table 2). In addition, the proportion of hospitalization days (differentially weighted whether at a regular ward or an intensive care unit) and the need for supportive therapies and care are noted (nutrition status and route, IgG substitution therapy, Table 2). Furthermore, "any other organ or immune dysfunction" and "any relevant chronic or recurring infestation or infection" are added to the score; the sum is then divided by the performance scale (Karnovsky or Lansky [28, 29]; see Table 2 for detailed calculation). Occurrence of a malignancy is noted separately but is not quantified within the numeric score. Parameters of the original version of the IDDA score were chosen to reflect the entire clinical spectrum of LRBA deficiency according to previously published case series and reviews. In the newly adapted version 2.1, only two major changes were introduced: the item 'hemophagocytosis' was added as additional possible feature of immune dysregulation to extend the utility to practically all PIRDs or IEI with immune dysregulation, and the calculation method (formula) was slightly modified to correct for very low performance scales (Table 2, bottom line). In contrast to the presented other 9 measures in IEI, many of which focus on disease-specific anatomical (HIES), infectious (CVID, and many others) or inflammatory (HLH, CVID, AIDAI) parameters, and, especially, to the other morbidity and disease activity measures, the IDDA2.1 score specifically addresses all most relevant features of immune dysregulation in IEI in a semiquantified, comprehensive, organspecific manner, and includes indicators of the actual disease and treatment burden, while maintaining relative simplicity. In fact, its composition is designed to allow documentation by retrospective chart review by a medical documentarist after a patient visit, as performed even as part of a registry study, without the need to obtain specific laboratory values or questionnaires, or to schedule specific imaging or physiological tests ahead. Assessing patient-reported symptom scores or quality of life/outcome measures by questionnaires (as in the AIDAI or P-CID studies, respectively) was omitted for the same reason as was the inclusion of results from particular functional, laboratory, or imaging analyses (as, e.g., in the CHAI score) - practicability. Nevertheless, recording the presence, severity, or absence of each parameter and the IDDA score sum over time and different treatment phases of a patient should enable to draw a wide-ranging, highly defined picture of the clinical status and course of a patient with a PIRD or IEI with immune dysregulation. Although relatively simple by design (grading from 0 to 4 , see above), limitations of the scoring were encountered, for example, quantifying the severity of infections. A molluscum or mucocutaneous HSV infection of a DOCK8-deficient patient will be assessed differently than in a patient with CVID, and a severe pneumococcal pneumonia of a hypogammaglobulinemic patient will be treated differently from an EBV-linked systemic lymphoproliferative and inflammatory response syndrome in CD27 deficiency, although all of them will be scored as $3^{\circ}$ or $4^{\circ}$ infection (range $0-4^{\circ}$ ) depending on treatment response, reversibility, and whether the event was life-threatening. Furthermore, difficulties may arise in the judgement, whether organ lesions are caused by an infection or by infection-triggered immune dysregulation and as such should be counted as organ involvement (e.g., lung or CNS granulomata) - the distinction of which may be easy in certain and difficult or impossible in other occasions. However, the general focus of graded organ involvement caused by immune dysregulation of the IDDA score sum and its longitudinal course are not affected by these differences.

The IDDA2.1 score may be used for a wide range of applications in clinical documentation. Supplementary Fig. 1 shows examples of the first version of the IDDA 
Table 2 The immune deficiency and dysregulation activity (IDDA2.1) score

\section{Parameters (for grading $0-4^{\circ}$, see below ${ }^{1}$; line titles serve as examples)}

Autoimmune (AI) cytopenia

Hemophagocytosis I HLH (according to clinical AND lab criteria of the HS)

Enteropathy I IBD

Lymphoproliferation I splenomegaly | hepatomegaly

Parenchymal lung disease I LIP I GLILD

Skin or eye manifestations I eczema, uveitis, alopecia, vitiligo, other

Granulomatous disease in any organ (except GLILD)

Endocrinopathy I IDDM, thyreoiditis, other

Arthritis I other musculoskeletal

AI-hepatitis I cholangitis I pancreatitis

Glomerulonephritis I nephropathy, tubulopathy

Neurologic manifestations of immune dysregulation I CNS autoimmunity, inflammation, vasculitis

Failure to thrive I malresorption, wasting

Severe infections I opportunistic (excl. chronic infestation, see below)

Other factors and symptoms (will multiply or add to the IDDAscore) ${ }^{2}$

15 Karnovsky / Lansky Performance Scale (\%)

16 Hospitalization

(days out of 100 days; including day clinic stays, excl. intensive care unit [ICU])

17 Mechanical ventilation or other ICU measures

(days out of 100 days; except elective procedures)

18 Immunoglobulin substitution therapy I hypogammaglobulinemia

19 Any relevant chronic or recurring infestation/infection (e.g., Norovirus, EBV...)

20 Any other organ dysfunction / malady (e.g., cardiomyopathy, kidney failure, allergies...)

$21 \quad$ Nutrition / dietary status and habits

22 Malignancy (separately noted, not added to numeric score)

Formula for IDDA2.1 score total (Excel® format)

$=($ SUM $($ line1:line14 $)+$ IF $($ line16 < 40;line16*0.1;4) + IF $($ line17 < 10;line17*0.8;8) + SUM $($ line18:lin e21))*IF(line15 > 29;150/line15;6)

${ }^{1}$, grading for lines 1-14: 0 , absent; 1 , mild, transient, not requiring treatment; 2 , moderate, intermittent therapy needed; 3 , severe, continuous therapy needed; 4 , life-threatening, refractory, irreversible

${ }^{2}$, lines $15-17$ are percentages, lines $18-21$ are scored as follows: line 18 (0, no; 2, sporadic; 3 [iv], regularly IVIG; 3[sc], regularly SCIG); line 19 (0, no; 1, asymptomatic infestation; 2, oligosymptomatic recurring infection; 3, recurring symptomatic infection requiring on/off treatment; 4, chronic infection requiring permanent treatment or refractory infection, only score worst if more than one microbial agents are relevant); line 20 (e.g., hepatopathy, cardiomyopathy, kidney failure; please quantify if possible: 0 , no organopathy; 1, mild transient dysfunction; 2, chronic mild dysfunction; 3, moderate-severe dysfunction; 4, clinically compromising dysfunction requiring treatment or replacement therapy, only score worst if more than one organ is involved); line 21 ( 0 , normal; 1 , modified disease-adjusted; 2 , part-formula medically advised; 3 , tube-feeding and/or full-formula or partial parenteral nutrition (irregularly); 4, total parenteral nutrition) score as it was used in a retrospective study of patients with LRBA deficiency that compared the clinical courses before, during, and after different treatments over time or in patients with residual LRBA protein expression as compared to those with absent LRBA protein [1], heatmaps illustrate the severity of each organ involvement before and after a certain therapeutic measure was applied, spaghetti plots may be used for longitudinal comparisons (Supplementary Fig. 1), and Kaplan-Meier curves may be plotted to compare outcomes of cohorts with different IDDA starting scores (not shown) [1].

A new accompanying feature that can be used to document the phenotype of patients or patient cohorts by using the IDDA2.1 score is the kaleidoscope function. It relies on the same data collected for the IDDA2.1 score, but instead of calculating a numerical score, the frequency of the presence of 17 of the 22 features/items within a patient cohort is plotted in a circular arrangement (spider or radar chart, listing the parameters in a fixed order as in Table 2), resulting in a recognizable, relatively pathwayspecific pattern. To illustrate this arrangement, we applied the IDDA kaleidoscope score to 18 different IEI, some of which shared the same impaired pathway (e.g., CTLA-4 haploinsufficiency, LRBA, and DEF6 deficiencies, see Fig. 2A; data are derived from published patient series or reviews that are cited in the legend). Others were chosen 
A
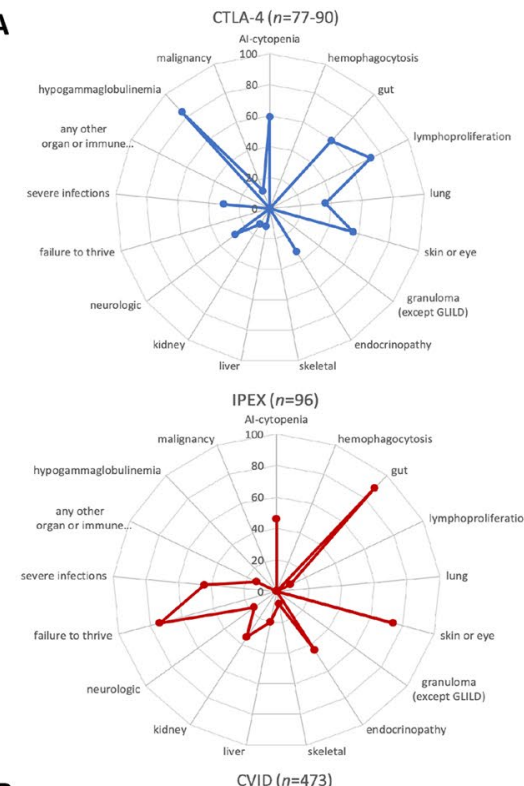

B
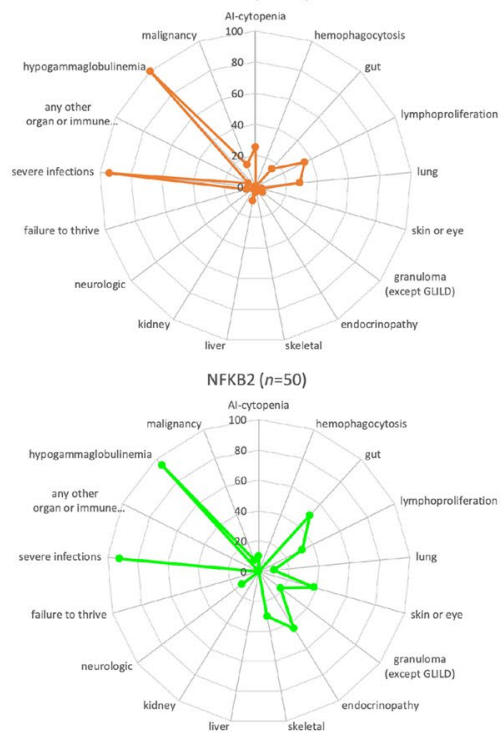

C

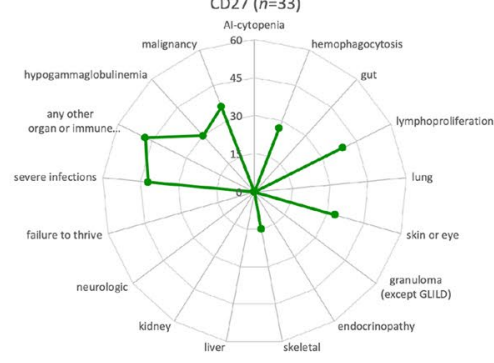

SH2D1A $(n=91-272)^{*}$

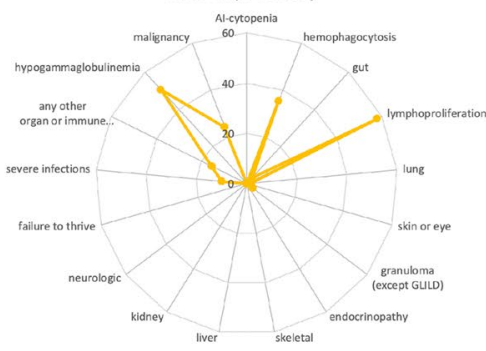

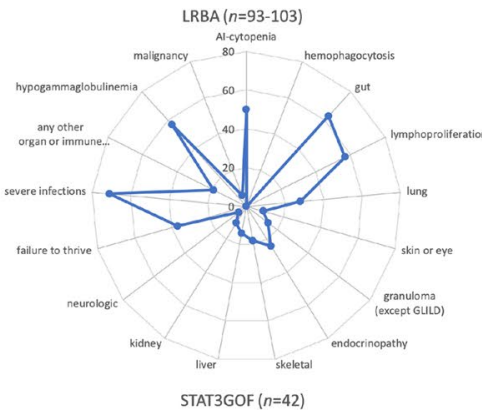
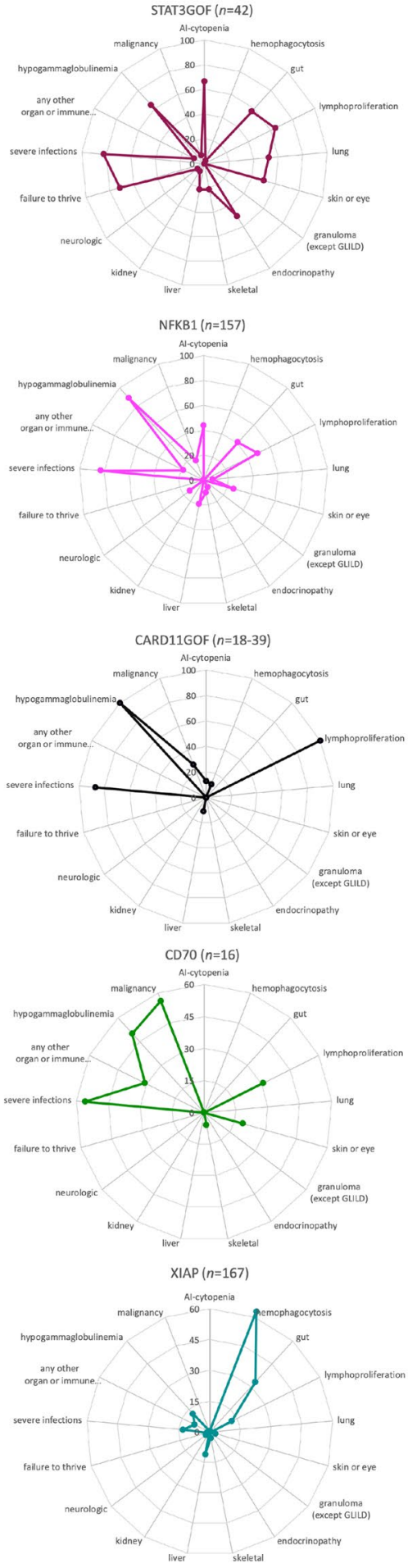
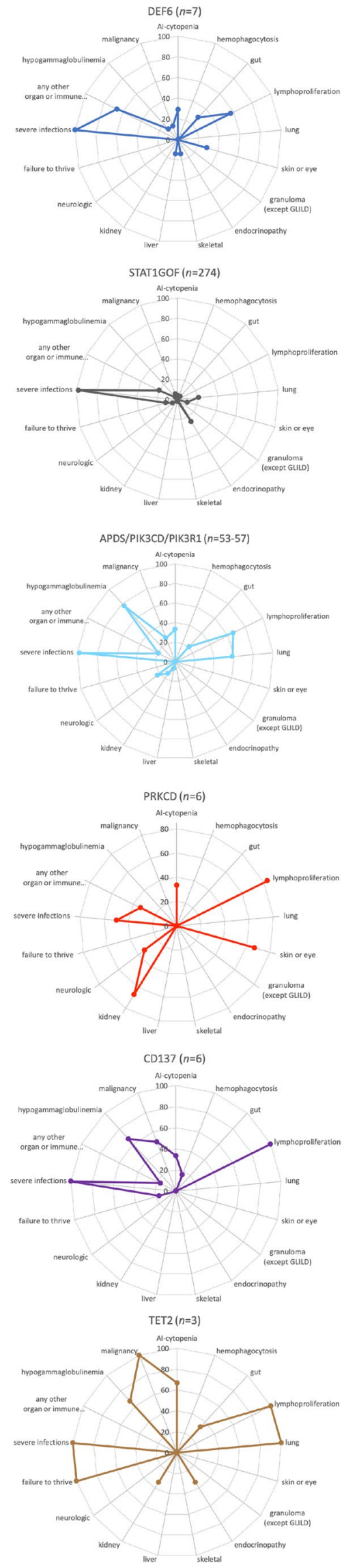
4Fig. 2 Immune deficiency and dysregulation phenotype patterns visualized by the IDDA2.1 kaleidoscope score for 18 exemplary IEI. The kaleidoscope function makes use of 17 out of 22 parameters documented in the IDDA score (terms \#1-14; \#20; \#18; and \#22, see Table 2), reduced to qualitative information about organ involvement and other features in a patient or a patient cohort, plotted according to the fixed order of parameters in a radar (spider) chart on 17 $y$-axes arranged in a circle. A Primary immune regulatory disorders, modified from [30] and supplemented with additional IEI, with data derived from reviews, case series, or large cohorts [1, 20, 31-36]; B Predominantly antibody deficiencies and combined immunodeficiencies [37-43]; C Diseases of immune regulation with EBV-susceptibility [44-50]. Data for part of the CARD11GOF and all of the XIAP plots were derived from unpublished data to appear in Hauck et al., 2021, and a manuscript in preparation by Yang and Burns et al., 2021, respectively. The patient numbers presented in the title of each plot may vary slightly regarding some features that were not available from all patients, but are always presented as a percentage on $17 y$-axes. In the regular (22-parametric) IDDA score originally developed for LRBA deficiency [1], each criterion is semi-quantified per patient from 0 to $4^{\circ}$, please refer to Table 2 for details. The fulllength $y$-axis titles are autoimmune (AI)-cytopenia; hemophagocytosis I HLH (according to clinical AND lab criteria of the Histiocyte Society); gut, enteropathy I IBD (inflammatory bowel disease); lymphoproliferation I splenomegaly I hepatomegaly; lung, parenchymal lung disease I LIP (lymphocytic interstitial pneumonitis)| GLILD (granulomatous lymphocytic interstitial lung disease); skin or eye manifestations I eczema, uveitis, alopecia, vitiligo, other; granulomatous disease in any organ (other than GLILD); endocrinopathy I IDDM (insulin-dependent diabetes mellitus), thyroiditis, other; skeletal, arthritis I other musculoskeletal manifestations; liver, AI-hepatitis I cholangitis I pancreatitis; kidney, glomerulonephritis I nephropathy, tubulopathy; neurologic manifestations; failure to thrive I malresorption, wasting; severe infections I severe or opportunistic infections (excl. asymptomatic chronic infestation; excluding "EBV-susceptibility"); any other organ or immune dysfunction/malady (e.g., cardiomyopathy, kidney failure, autoinflammation, allergy); hypogammaglobulinemia and/or immunoglobulin substitution therapy; malignancy, lymphoma (separately added to IDDA score, not included in the score calculation); *, the footnote (asterisk) in SH2DA1 deficiency (XLP1) should indicate that, although this topic is debatable, the liver and kidney involvement in fulminant infectious mononucleosis was not counted under immune dysregulation (\#10-11), likewise the CNS involvement in patients with HLH and XLP1 was not counted as organ-specific immune dysregulation (\#12), and aplastic anemia observed in patients with XLP1 was not counted as autoimmune cytopenia (\#1) to distinguish their pathogenesis from "primarily" immune-mediated organ manifestations in PIRDs

due to their frequency or their novelty. For educational purposes, we grouped the 18 IEI into predominantly regulatory $\mathrm{T}$ cell disorders (Tregopathies), CVID)/CVID-like disorders, and PIRDs with EBV susceptibility (Fig. 3A, B, and $\mathrm{C}$, respectively). Of note, the kaleidoscope patterns of CTLA-4 haploinsufficiency, LRBA, and DEF6 deficiencies look very similar, but differ from those of IPEX syndrome or CVID (Fig. 3A, B). On the other hand, the patterns for CVID, NFKB1, and NFKB2 deficiency resemble each other closely (Fig. 3B), possibly reflecting a large proportion of undetected NFKB1 and NFKB2 deficiencies among CVID cohorts, as do the patterns for the receptor-ligand pair CD27 and CD70 deficiencies (Fig. 3C). Expectedly, the 'kaleidoscope pattern' of XLP1 differs from that of XLP2/XIAP deficiency, whereas, independent of the direct pathomechanism, that of STAT3GOF looks very much alike that of LRBA deficiency. Thus, an immediate conclusion of a similarity of the biomechanistic basis and thus treatment recommendation cannot be drawn from this first, retrospectively generated plot. An alternative way to present these data could be a heatmap (Supplementary Fig. 2), which may be preferred for pattern recognition by people used to interpret array data. To verify the grouping of IEI based on clinical experience and to prioritize the clinical features according to their relevance in this set of 18 IEI, we performed an unsupervised hierarchical clustering analysis of the same data which yielded clusters of diseases according to their feature frequency profiles (neighbors in the horizontal order and clustering, Fig. 3) that is strikingly similar but not identical to that of our clinical and "visual" order applied in the spider charts shown in Fig. 2. The heatmap derived from this clustering analysis reveals "pedigrees" of the most relevant and discriminative clinical parameters among the IDDA2.1 list for this set of 18 IEI (vertical order/clustering, Fig. 3). Because we used aggregated cohort data from large series or reviews to generate Fig. 2 and lack most of the raw (single patient) data, we were unable to calculate similarities of the kaleidoscope patterns statistically, e.g., in principal component analyses. Although individual patients will, naturally, be unlikely to resemble complete congruence in these phenotype expression analyses from aggregate data, the IDDA2.1 kaleidoscope function may gain importance if applied regularly and fed into a machine learning algorithm as discussed below. For educational purposes, the present catalogue of spider charts or heatmaps may be extended to any IEI with immune dysregulation.

\section{Conclusions and Future Perspectives}

A standardized, quantitative definition of the clinical phenotype of an IEI in its entirety and its consequences on the patient's quality of life, need for supportive care, and disease burden is relevant both in disease- or drug-specific studies as well as in prospective patient registry studies to learn about the natural course of the disease.

Ensuring the comparability and transferability of scoring results between specific disorders and between patients with undiagnosed IEI, either cross-sectionally or over time, is an advantage of more general disease scores as opposed to single-disease-focused scores. However, in order to propose a clinical score that covers more than one distinct disease and likewise measures disease activity, several obstacles must first be overcome. First, the balance has to be maintained between simplicity and feasibility, on the one 
Fig. 3 'Phenotype expression array' showing results of unsupervised hierarchical clustering of 18 IEI with immune dysregulation according to the IDDA2.1 parameter list. The same patient cohorts were analyzed as shown and referenced in Fig. 2. The clustered heatmap was created by using the $\mathrm{R}$ package pheatmap 1.0.12 (Raivo Kolde, 2019). The hierarchical clustering was the result of complete linkage based on Euclidean distances (after logistic transformation truncated at $1 \%$ ). Red boxes indicate high frequency, blue boxes low frequency. "Pedigrees" indicate the calculated similarities or distances; not taking into account the relative frequency of patients evaluated in the original data collection (see numbers next to the IEI diagnosis). Therefore, due to very low patient numbers $(<10)$, phenotype data for DEF6, PRKCD, CD137, and TET2 deficiencies are less reliable than those for other IEI with higher patient numbers

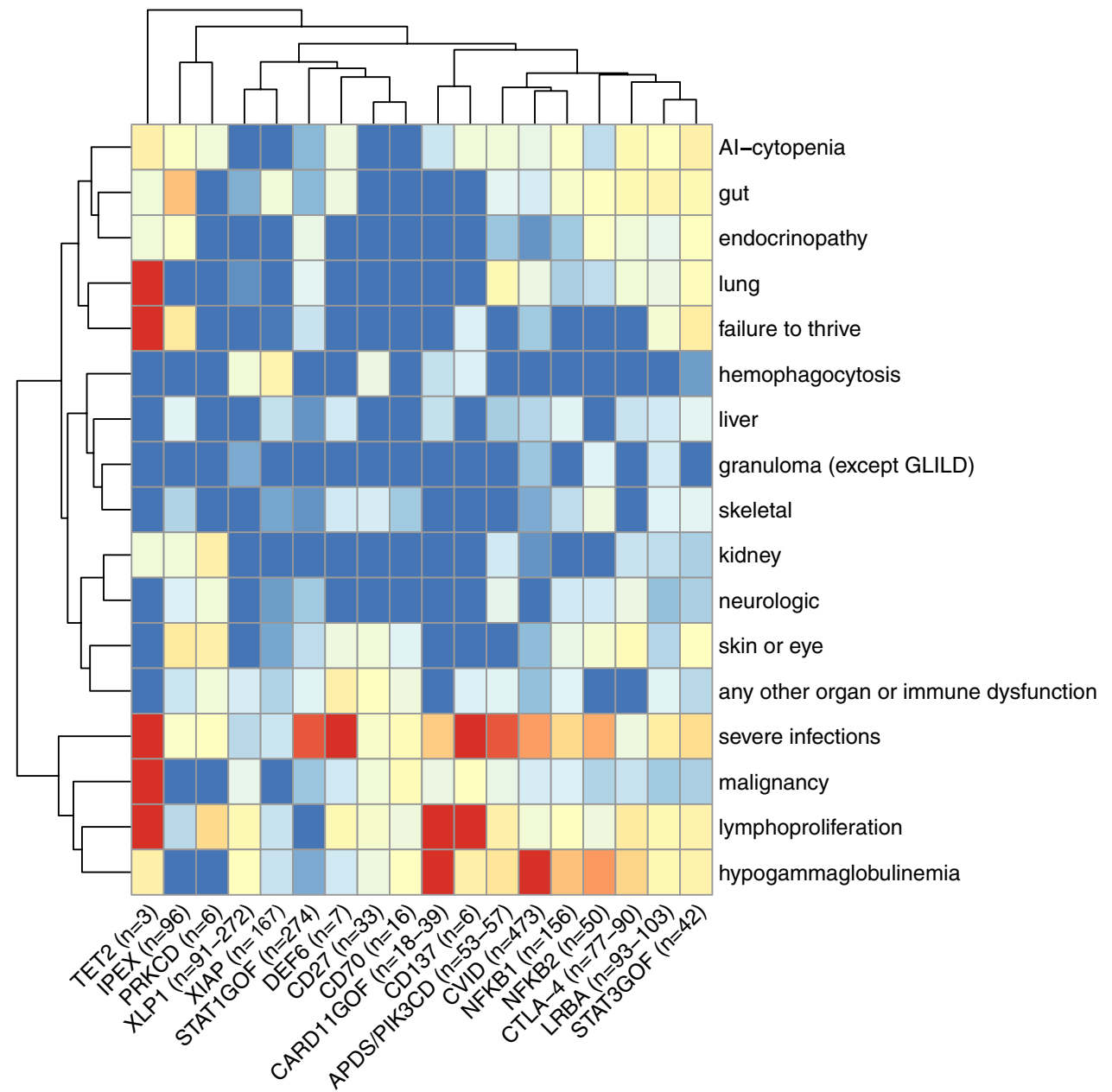

hand, and detail and specificity on the other hand, while still avoiding the need to refer to a meticulous handbook or user manual. It is difficult to define which organ damage should be included into the immune dysregulationdependent scoring process, and which should be included only into "other organ dysfunction," e.g., the differentiation between whether parenchymal changes in an organ should be attributed to recurring invasive infections or represent the result of inflammation and dysregulated organ infiltration. The same vagueness applies to the different reasons of lymphoproliferation whether "genuine" (e.g., in autoimmune lymphoproliferative syndrome) or as result of chronic lymphadenopathy, furthermore, to hepatosplenomegaly in the context of HLH or CVID, or to cytopenia associated with splenomegaly, etc. (see also the asterisk-marked footnote at XLP1 in Fig. 3C). Similarly, any definitions may not always be uniformly understandable or may show varying levels of resolution, e.g., the terms "autoimmunity" or "cytopenia" may not be very helpful, given the fact that either the target organ or the pathomechanism remained undefined, thus impairing the correct scoring. Furthermore, user-dependent factors, such as variations in the assessment of disease severity, may compromise the comparability of data. Second, the phenotype of a specific IEI with a typically increased vulnerability in a defined organ (e.g., colitis), towards certain infectious microorganisms (e.g., herpes viruses, mycobacteria, candidiasis), or with another characteristic clinical feature (e.g., allergy, vasculitis) may not be depicted proportionally through the use of a more general score such as the IDDA score. In these cases, a disease-specific score may yield more granular results and thus be more adequately applied or it should be used in addition to a standard score. Third, the disease activity depends on the treatment status of a patient. Therefore, documenting a score of the above-mentioned category 2 (severity/disease activity), such as the IDDA score, should automatically involve its timely correlation with the current therapy of a patient and ideally be repeated at regular intervals.

One main goal of the human phenotype ontology (HPO) project is to standardize the terminology used to describe phenotypic characteristics, the definitions of disease features and manifestations, symptoms, laboratory abnormalities, and other clinical aspects. This project has recently been 
extended to include many IEI [51-55]. An initial attempt to include HPO terms into the IDDA score was temporarily aborted, because we aimed to achieve the utmost simplicity when incorporating the 22 parameters into the ESID registry database. Nevertheless, the inclusion of HPO codes into the background of data entry fields, possibly with refined subitems, might increase the accuracy and resolution of clinical data documentation. An international initiative is ongoing to define these terms in more detail and to extend the applicability of HPO terms in IEI [54].

In the AIDAI, for example, significant efforts have been made to achieve statistical validation [21], and this validation might be achieved with respect to other scores as well. However, the composition of a score in IEI will mostly be based on clinical observations of the two- or three-digit patient numbers only, and its application will be used for descriptive purposes rather than for strictly exclusive decision-making or stratifications. Because a clinical score in IEI will never be a stand-alone criterion for making or excluding a diagnosis or a treatment decision, and because largely overlapping phenotypes are observed in many of these extremely rare diseases, it will be difficult to provide statistical validation for many of the purposes these scores may fulfill. With regard to the IDDA2.1 score, an open science prospective study proposal has been approved and launched in the context of the ESID registry.

We propose that, collecting IDDA2.1 score data and applying the IDDA kaleidoscope function in a standardized manner in patients with PIRDs in the future may allow validation of pattern comparisons, algorithm-assisted recognition, and potentially even phenotype-based diagnosis prediction of PIRDs. To this end, the IDDA2.1 score has been implemented in the European Society for Immunodeficiencies (ESID) registry database [56] as an optional module to serve as a basis for a prospective study using monogenic IEIs as a learning cohorts. With enough data from known IEIs and, ideally, multiple patient time points under different therapies, this should allow to generate a machine-learning-powered tool which should eventually assist clinicians in making diagnoses, monitor expectable risk organs, and support phenotype-driven, "semitargeted" treatment decisions for immune dysregulation in undiagnosed patients. In this study, attempts will be made to rank the relevance of items collected in the ESID registry according to their predictive power regarding the diagnosis, progression, and treatment response, e.g., by applying logistic regression models, time-dependent Coxregression models, and the nomograms based on these, as described elsewhere [57, 58]. This should enable the ESID registry's composition and application to be further strengthened and refined. When taking this approach, we will apply unsupervised machine learning algorithms to detect similarities in patterns in training cohorts consisting of patients with known monogenic IEI.

If scores in IEI are regarded as assistive measures and their application is restricted to specific studies or in specific institutions consistently, they may be very helpful in clinical and clinical research practice. Using and applying a "score standard" in the clinical practice in IEI, as shown with the IDDA2.1 score for PIRDs and other IEI with immune dysregulation, may prove useful as an educational tool and enhance the awareness of the complexity of phenotypes and complications, ultimately improving patient outcomes.

Supplementary Information The online version contains supplementary material available at https://doi.org/10.1007/s10875-021-01177-2.

Acknowledgements The authors thank Dr. Diether Kramer, Graz, for fruitful discussions about future biomathematical research options, Dr. Bodo Grimbacher, Freiburg, for his expert advice on historical and current clinical scores in IEI, Dr. Siobhan Burns for sharing data on a large retrospective data analysis for XIAP deficiency before their publication, and Dr. Sara Crockett (www.saras-science.com; Graz) for providing scientific language editing services.

Author Contribution MGS created the immune deficiency and dysregulation activity (IDDA score) and its kaleidoscope function, wrote the manuscript, and designed the figures. VKT and ALH collected the data and drafted the tables. LY and FH provided phenotypic patient cohort data on XIAP deficiency and CARD11GOF, respectively. MS provided advice about statistical and computer-assisted methods. FQ performed hierarchical cluster analyses and designed Fig. 3. MB tested the consistency of the data interpretation and provided advice about its presentation. All authors revised the manuscript.

Funding Open access funding provided by Medical University of Graz. MGS was in part funded by the Styrian Children's Cancer Aid foundation (Steirische Kinderkrebshilfe). FH was funded the the Else Kröner-Fresenius Stiftung (EKFS, 2017_A110) and the German Federal Ministry of Education and Research (BMBF, 01GM1910C).

Data Availability Not applicable.

Code Availability Not applicable.

\section{Declarations}

Ethics Approval Not applicable.

Consent to Participate Not applicable.

Consent for Publication Not applicable.

Conflict of Interest The authors declare no competing interests.

Open Access This article is licensed under a Creative Commons Attribution 4.0 International License, which permits use, sharing, adaptation, distribution and reproduction in any medium or format, as long as you give appropriate credit to the original author(s) and the source, provide a link to the Creative Commons licence, and indicate if changes were made. The images or other third party material in this article are 
included in the article's Creative Commons licence, unless indicated otherwise in a credit line to the material. If material is not included in the article's Creative Commons licence and your intended use is not permitted by statutory regulation or exceeds the permitted use, you will need to obtain permission directly from the copyright holder. To view a copy of this licence, visit http://creativecommons.org/licenses/by/4.0/.

\section{References}

1. Tesch VK, Abolhassani H, Shadur B, Zobel J, Mareika Y, Sharapova $\mathrm{S}$, et al. Long-term outcome of LRBA deficiency in 76 patients after various treatment modalities as evaluated by the immune deficiency and dysregulation activity (IDDA) score. J Allergy Clin Immunol. 2020;145(5):1452-63.

2. Tangye SG, Al-Herz W, Bousfiha A, Chatila T, CunninghamRundles C, Etzioni A, et al. Human inborn errors of immunity: 2019 update on the Classification from the International Union of Immunological Societies Expert Committee. J Clin Immunol. 2020;40(1):24-64.

3. Bousfiha A, Jeddane L, Picard C, Al-Herz W, Ailal F, Chatila T, et al. Human inborn errors of immunity: 2019 update of the IUIS Phenotypical Classification. J Clin Immunol. 2020;40(1):66-81.

4. Grimbacher B, Schaffer AA, Holland SM, Davis J, Gallin JI, Malech HL, et al. Genetic linkage of hyper-IgE syndrome to chromosome 4. Am J Hum Genet. 1999;65(3):735-44.

5. Woellner C, Gertz EM, Schaffer AA, Lagos M, Perro M, Glocker EO, et al. Mutations in STAT3 and diagnostic guidelines for hyperIgE syndrome. J Allergy Clin Immunol. 2010;125(2):424-32e8.

6. Hejblum G, Lambotte O, Galicier L, Coppo P, Marzac C, Aumont $\mathrm{C}$, et al. A web-based delphi study for eliciting helpful criteria in the positive diagnosis of hemophagocytic syndrome in adult patients. PloS one. 2014;9(4):e94024.

7. Fardet L, Galicier L, Lambotte O, Marzac C, Aumont C, Chahwan $\mathrm{D}$, et al. Development and validation of the HScore, a score for the diagnosis of reactive hemophagocytic syndrome. Arthritis Rheumatol. 2014;66(9):2613-20.

8. Henter JI, Horne A, Arico M, Egeler RM, Filipovich AH, Imashuku S, et al. HLH-2004: Diagnostic and therapeutic guidelines for hemophagocytic lymphohistiocytosis. Pediatr Blood Cancer. 2007;48(2):124-31.

9. Engelhardt KR, McGhee S, Winkler S, Sassi A, Woellner C, Lopez-Herrera $\mathrm{G}$, et al. Large deletions and point mutations involving the dedicator of cytokinesis 8 (DOCK8) in the autosomal-recessive form of hyper-IgE syndrome. J Allergy Clin Immunol. 2009;124(6):1289-302e4.

10. Fukaya S, Yasuda S, Hashimoto T, Oku K, Kataoka H, Horita T, et al. Clinical features of haemophagocytic syndrome in patients with systemic autoimmune diseases: analysis of 30 cases. Rheumatology (Oxford). 2008;47(11):1686-91.

11. Ravelli A, Magni-Manzoni S, Pistorio A, Besana C, Foti T, Ruperto N, et al. Preliminary diagnostic guidelines for macrophage activation syndrome complicating systemic juvenile idiopathic arthritis. J Pediatr. 2005;146(5):598-604.

12. Albert MH, Notarangelo LD, Ochs HD. Clinical spectrum, pathophysiology and treatment of the Wiskott-Aldrich syndrome. Curr Opin Hematol. 2011;18(1):42-8.

13. Ochs HD. Wiskott-Aldrich Syndrome. 2021 [cited May 24, 2021]. In: UpToDate ${ }^{\circledR}$ [Internet]. Waltham, MA: Wolters Kluwer, [cited May 24, 2021].

14. Ochs HD, Thrasher AJ. The Wiskott-Aldrich syndrome. J Allergy Clin Immunol. 2006;117(4):725-38 (quiz 39).
15. Albert MH, Freeman AF. Wiskott-Aldrich Syndrome (WAS) and dedicator of cytokinesis 8- (DOCK8) deficiency. Front Pediatr. 2019;7:451.

16. Yong PF, Thaventhiran JE, Grimbacher B. "A rose is a rose is a rose," but CVID is Not CVID common variable immune deficiency (CVID), what do we know in 2011? Adv Immunol. 2011;111:47-107.

17. Mokhtari M, Shakeri A, Mirminachi B, Abolhassani H, Yazdani $\mathrm{R}$, Grimbacher B, et al. Important factors influencing severity of common variable immunodeficiency. Arch Iran Med. 2016;19(8):544-50.

18. Ameratunga R. Assessing disease severity in common variable immunodeficiency disorders (CVID) and CVID-like disorders. Front Immunol. 2018;9:2130.

19. Speckmann C, Doerken S, Aiuti A, Albert MH, Al-Herz W, Allende LM, et al. A prospective study on the natural history of patients with profound combined immunodeficiency: an interim analysis. J Allergy Clin Immunol. 2017;139(4):1302-10.e4.

20. Barzaghi F, Amaya Hernandez LC, Neven B, Ricci S, Kucuk $\mathrm{ZY}$, Bleesing JJ, et al. Long-term follow-up of IPEX syndrome patients after different therapeutic strategies: an international multicenter retrospective study. J Allergy Clin Immunol. 2018;141(3):1036-49e5.

21. Piram M, Kone-Paut I, Lachmann HJ, Frenkel J, Ozen S, Kuemmerle-Deschner J, et al. Validation of the auto-inflammatory diseases activity index (AIDAI) for hereditary recurrent fever syndromes. Ann Rheum Dis. 2014;73(12):2168-73.

22. Piram M, Frenkel J, Gattorno M, Ozen S, Lachmann HJ, Goldbach-Mansky R, et al. A preliminary score for the assessment of disease activity in hereditary recurrent fevers: results from the AIDAI (Auto-Inflammatory Diseases Activity Index) Consensus Conference. Ann Rheum Dis. 2011;70(2):309-14.

23. Stephenson C, Prajapati VH, Hunter C, Miettunen P. Novel use of Autoinflammatory Diseases Activity Index (AIDAI) captures skin and extracutaneous features to help manage pediatric DITRA: a case report and a proposal for a modified disease activity index in autoinflammatory keratinization disorders. Pediatr Dermatol. 2020;37(4):670-6.

24. Therapeutic Use of Tadekinig Alfa in NLRC4 Mutation and XIAP Deficiency as Open Label Extension [Internet]. https://www.clini caltrials.gov: NCT03512314. 2018-2023. Accessed 17 Nov 2021

25. Agarwal S, Cunningham-Rundles C. Treatment of hypogammaglobulinemia in adults: a scoring system to guide decisions on immunoglobulin replacement. J Allergy Clin Immunol. 2013;131(6):1699-701.

26. Hanitsch L, Baumann U, Boztug K, Burkhard-Meier U, Fasshauer $\mathrm{M}$, Habermehl P, et al. Treatment and management of primary antibody deficiency: German interdisciplinary evidence-based consensus guideline. Eur J Immunol. 2020;50(10):1432-46.

27. Krudewig J, Baumann U, von Bernuth H, Borte M, BurkhardMeier U, Dueckers G, et al. Interdisciplinary AWMF guideline for the treatment of primary antibody deficiencies. Klin Padiatr. 2012;224(6):404-15.

28. Karnofsky DA, Burchenal JH. The clinical evaluation of chemotherapeutic agents in cancer. In: MacLeod C (editor),Evaluation of Chemotherapeutic Agents. New York: Columbia University Press; 1949. pp. 169.

29. Lansky SB, List MA, Lansky LL, Ritter-Sterr C, Miller DR. The measurement of performance in childhood cancer patients. Cancer. 1987;60(7):1651-6.

30. Gamez-Diaz L, Seidel MG. Different apples, same tree: visualizing current biological and clinical insights into CTLA-4 insufficiency and LRBA and DEF6 deficiencies. Front Pediatr. 2021;9:662645.

31. Schwab C, Gabrysch A, Olbrich P, Patino V, Warnatz K, Wolff $\mathrm{D}$, et al. Phenotype, penetrance, and treatment of 133 cytotoxic 
T-lymphocyte antigen 4-insufficient subjects. J Allergy Clin Immunol. 2018;142(6):1932-46.

32. Gamez-Diaz L, August D, Stepensky P, Revel-Vilk S, Seidel MG, Noriko M, et al. The extended phenotype of LPS-responsive beige-like anchor protein (LRBA) deficiency. J Allergy Clin Immunol. 2016;137(1):223-30.

33. Habibi S, Zaki-Dizaji M, Rafiemanesh H, Lo B, Jamee M, Gamez-Diaz L, et al. Clinical, immunologic, and molecular spectrum of patients with LPS-responsive beige-like anchor protein deficiency: a systematic review. J Allergy Clin Immunol Pract. 2019;7(7):2379-86e5.

34. Serwas NK, Hoeger B, Ardy RC, Stulz SV, Sui Z, Memaran N, et al. Human DEF6 deficiency underlies an immunodeficiency syndrome with systemic autoimmunity and aberrant CTLA-4 homeostasis. Nat Commun. 2019;10(1):3106.

35. Fournier B, Tusseau M, Villard M, Malcus C, Chopin E, Martin E, et al. DEF6 deficiency, a mendelian susceptibility to EBV infection, lymphoma, and autoimmunity. J Allergy Clin Immunol. 2021;147(2):740-3e9.

36. Toubiana J, Okada S, Hiller J, Oleastro M, Lagos Gomez M, Aldave Becerra JC, et al. Heterozygous STAT1 gain-of-function mutations underlie an unexpectedly broad clinical phenotype. Blood. 2016;127(25):3154-64.

37. Lorenzini T, Fliegauf M, Klammer N, Frede N, Proietti M, Bulashevska A, et al. Characterization of the clinical and immunologic phenotype and management of 157 individuals with 56 distinct heterozygous NFKB1 mutations. J Allergy Clin Immunol. 2020;146(4):901-11.

38. Resnick ES, Moshier EL, Godbold JH, Cunningham-Rundles C. Morbidity and mortality in common variable immune deficiency over 4 decades. Blood. 2012;119(7):1650-7.

39. Coulter TI, Chandra A, Bacon CM, Babar J, Curtis J, Screaton N, et al. Clinical spectrum and features of activated phosphoinositide 3-kinase delta syndrome: a large patient cohort study. J Allergy Clin Immunol. 2017;139(2):597-6064.

40. Dimitrova D, Nademi Z, Maccari ME, Ehl S, Uzel G, Tomoda T, et al. International retrospective study of allogeneic hematopoietic cell transplantation for activated PI3K-delta syndrome. J Allergy Clin Immunol (ahead of print). 2021;S0091-6749(21)00810-1. https://doi.org/10.1016/j.jaci.2021.04.036. Online ahead of print.

41. Klemann C, Camacho-Ordonez N, Yang L, Eskandarian Z, RojasRestrepo JL, Frede N, et al. Clinical and immunological phenotype of patients with primary immunodeficiency due to damaging mutations in NFKB2. Front Immunol. 2019;10:297.

42. Lu HY, Bauman BM, Arjunaraja S, Dorjbal B, Milner JD, Snow AL, et al. The CBM-opathies-a rapidly expanding spectrum of human inborn errors of immunity caused by mutations in the CARD11-BCL10-MALT1 complex. Front Immunol. 2018;9:2078.

43. Salzer E, Santos-Valente E, Keller B, Warnatz K, Boztug K. Protein kinase $\mathrm{C}$ delta: a gatekeeper of immune homeostasis. J Clin Immunol. 2016;36(7):631-40.

44. Alkhairy OK, Perez-Becker R, Driessen GJ, Abolhassani H, van Montfrans J, Borte S, et al. Novel mutations in TNFRSF7/CD27: clinical, immunologic, and genetic characterization of human CD27 deficiency. J Allergy Clin Immunol. 2015;136(3):703-+.

45. Ghosh S, Kostel Bal S, Edwards ESJ, Pillay B, Jimenez Heredia R, ErolCipe F, et al. Extended clinical and immunological phenotype and transplant outcome in CD27 and CD70 deficiency. Blood. 2020;136(23):2638-55.

46. Somekh I, Thian M, Medgyesi D, Gulez N, Magg T, Gallon Duque A, et al. CD137 deficiency causes immune dysregulation with predisposition to lymphomagenesis. Blood. 2019;134(18):1510-6.

47. Alosaimi MF, Hoenig M, Jaber F, Platt CD, Jones J, Wallace $\mathrm{J}$, et al. Immunodeficiency and EBV-induced lymphoproliferation caused by 4-1BB deficiency. J Allergy Clin Immunol. 2019;144(2):574-83e5.

48. Booth C, Gilmour KC, Veys P, Gennery AR, Slatter MA, Chapel $\mathrm{H}$, et al. X-linked lymphoproliferative disease due to SAP/SH2D1A deficiency: a multicenter study on the manifestations, management and outcome of the disease. Blood. 2011;117(1):53-62.

49. Seemayer TA, Gross TG, Egeler RM, Pirruccello SJ, Davis JR, Kelly CM, et al. X-linked lymphoproliferative disease: twenty-five years after the discovery. Pediatr Res. 1995;38(4):471-8.

50. StremenovaSpegarova J, Lawless D, Mohamad SMB, Engelhardt KR, Doody G, Shrimpton J, et al. Germline TET2 loss of function causes childhood immunodeficiency and lymphoma. Blood. 2020;136(9):1055-66.

51. Gasteiger LM, Robinson PN, Pazmandi J, Boztug K, Seppanen MRJ, Seidel MG, et al. Supplementation of the ESID registry working definitions for the clinical diagnosis of inborn errors of immunity with encoded human phenotype ontology (HPO) terms. J Allergy Clin Immunol Pract. 2020;8(5):1778.

52. Kohler S, Carmody L, Vasilevsky N, Jacobsen JOB, Danis D, Gourdine JP, et al. Expansion of the Human Phenotype Ontology (HPO) knowledge base and resources. Nucleic Acids Res. 2019;47(D1):D1018-27.

53. Robinson PN, Kohler S, Bauer S, Seelow D, Horn D, Mundlos S. The Human Phenotype Ontology: a tool for annotating and analyzing human hereditary disease. Am J Hum Genet. 2008;83(5):610-5.

54. Haimel M, Pazmandi J, Heredia RJ, Dmytrus J, Bal SK, Zoghi $\mathrm{S}$, et al. Curation and expansion of human phenotype ontology for defined groups of inborn errors of immunity. J Allergy Clin Immunol. 2021:S0091-6749(21)00732-6. https://doi.org/10. 1016/j.jaci.2021.04.033.

55. Kohler S, Gargano M, Matentzoglu N, Carmody LC, Lewis-Smith D, Vasilevsky NA, et al. The Human Phenotype Ontology in 2021. Nucleic Acids Res. 2021;49(D1):D1207-17.

56. ESID. Online Registry: European Society for Immunodeficiencies - Registry Working Party; 2021 [Available from: https:// esid.org/Working-Parties/Registry-Working-Party/ESID-Regis try. Accessed 17 Nov 2021

57. Zhao W, He Z, Li Y, Jia H, Chen M, Gu X, et al. Nomogram-based parameters to predict overall survival in a real-world advanced cancer population undergoing palliative care. BMC Palliat Care. 2019;18(1):47.

58. Balachandran VP, Gonen M, Smith JJ, DeMatteo RP. Nomograms in oncology: more than meets the eye. Lancet Oncol. 2015;16(4):e173-80.

Publisher's Note Springer Nature remains neutral with regard to jurisdictional claims in published maps and institutional affiliations. 